\title{
AN EXTENDED SCHEME OF SPECTRAL CLASSIFICATION FOR OBJECTIVE PRISM SPECTRA
}

\author{
P.A. HANTZIOS ${ }^{1}$, E. KONTIZAS ${ }^{1}$, F. PASIAN ${ }^{2}$, A. DAPERGOLAS ${ }^{1}$, M. \\ KONTIZAS $^{3}$ and R. SMAREGLIA ${ }^{2}$ \\ ${ }^{1}$ Astronomical Institute, National Observatory of Athens, P.O. Box 20048, \\ GR 11810, Thission, Athens, GREECE. \\ ${ }^{2}$ Osservatorio Astronomico di Trieste, Via G.B. Tiepolo 11, 34131 Trieste, Italy. \\ ${ }^{3}$ Section of Astrophysics, Astronomy and Mechanics, Dept. of Physics, \\ University of Athens, Panepistimiopolis, GR 15783, Zographou, Athens, GREECE.
}

\begin{abstract}
A spectral classification scheme has been developed for objective prism spectra of LMC stars, taken with the $1.2 \mathrm{~m}$ UK Schmidt Telescope in Australia. Various line ratios, discontinuities, and continuum intensities have been used as criteria. The scheme is a 2-D system (spectral type - luminosity class), and the criteria are given in graphical form. The typical accuracy of our scheme is 2 subdivisions of spectral type. Comparison of our types with those given by other authors for common stars yields a very good correlation with deviations within our accuracy.
\end{abstract}

\section{Introduction - Observational Material}

High quality film copies of objective prism spectra (dispersion: $830 \AA / \mathrm{mm}$ ) on IIIa-J plates taken with the $1.2 \mathrm{~m}$ UKST were used for this work. The spectra were studied in the form of tracings taken by the PDS 1010A in the Trieste Astronomical Observatory, Italy, covering the spectral region from 3400 to $5400 \mathrm{~A}$. Our sample consists of 259 spectra of stars belonging to clusters, mainly in fields of the LMC, evenly distributed over spectral types and representing luminosity classes Ib, II, and early III. A typical spectrum of our sample is shown in Fig. 1. We use 39 criteria chosen among a great variety of criteria adopted previously for spectra of comparable resolution (Seitter 1975; Savage et al. 1985). The purpose of this work is to improve on the classification with this type of spectra over what has been done previously (Dapergolas et al. 1986; Kontizas et al. 1986) via a quantitative classification scheme, in order to study the stellar content of the LMC and the SMC in more detail.

\section{Spectral Classification}

The classification of stellar spectra is based on 39 criteria. The line ratios used are shown in Table 1, together with the Balmer discontinuity, the CaII H-K discontinuity, and the intensities at four selected wavelengths $(5100,4200,3600$, and 3420A) together with their differences. The line ratios mentioned above are not pure ratios, but they include weaker neighbouring lines too due to our resolution. We use our criteria in the form of diagrams of the type: criterion vs. spectral type for different luminosity classes wherever it was found so. They are presented in the 


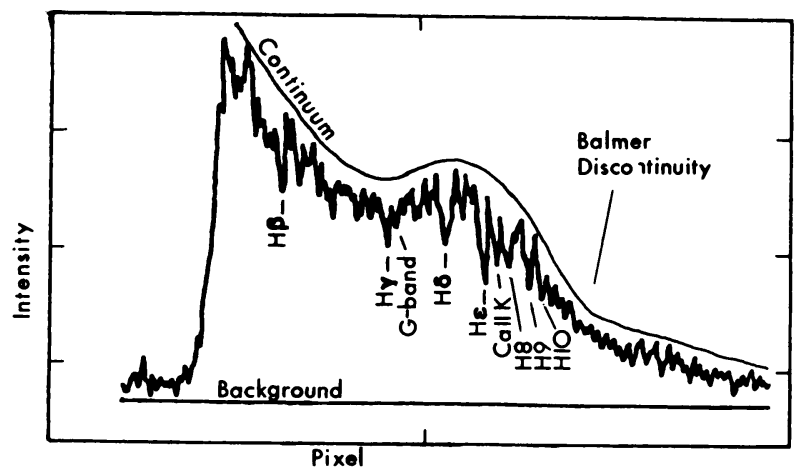

Figure 1. The spectrum of an A7 II star.

original extended form of this paper (Hantzios et al. 1993). The criteria are assigned statistical weights for the various spectral types and luminosity classes, and the calculated spectral type of a star is the weighted average of all criteria present in its spectrum. The typical accuracy of our scheme is 2 spectral subtypes and 1 luminosity subclass.

Table 1. Line ratios used in the classification

\begin{tabular}{|c|c|c|}
\hline HeII686/HeI4471 & CI4268/FeI4233 & FeI4144/Hס \\
\hline HeII4686/H及 & HeI3634/ZrII+VII3557 & CrI-FeI4272-75/H $\gamma$ \\
\hline $\mathrm{HeI} 4471 / \mathrm{H} \gamma$ & H9+MgI3829-38/H8 & TiO4955/TiO4626 \\
\hline HeI4922/Hß & $\mathrm{H} 8 / \mathrm{K}$ & TiO4804/TiO4626 \\
\hline $\mathrm{HeI} 4388 / \mathrm{H} \gamma$ & $\mathbf{K} / \mathbf{H}+\mathbf{H} \boldsymbol{\varepsilon}$ & FeI+TiO4435-67/FeI4383 \\
\hline HeI4144/Hठ & $\mathbf{G} / \mathbf{H} \boldsymbol{\gamma}$ & TiO4422/FeI4383 \\
\hline HeI4026/Hع & FeI-SrII4064-78/H $\delta$ & TiO4669/FeI4383 \\
\hline HeI3926/H8 & FeI-SrII-CN4173-4216/Hס & TiO4669/CaI4227 \\
\hline HeI3820/H9 & FeI4383/H $\gamma$ & TiO4761/FeI4383 \\
\hline HeI4144/FeII4179 & FeI-SrII-CN4173-4216/G & $\mathrm{CaI} 4227 / \mathrm{G}$ \\
\hline
\end{tabular}

\section{Evaluation and Conclusions}

Our accuracy is limited by the dispersion of the spectra. In general, for the bright spectra we have good accuracy while for the faint ones we have large deviations. Other uncertainty factors are continuum uncertainty, mis-identification of spectral lines, overlapping spectra, and off-axis scanning. All these result in an average uncertainty of 2 spectral subtypes. 27 stars of our sample have been classified by other authors as well. The comparison of our results yields a linear correlation with no significant systematic deviations. The standard deviation of this correlation is 3 subtypes. We conclude that our scheme with its improved accuracy relative to previous work is appropriate for examining the great amounts of data available and we expect to find more details about the stellar content, age, evolution, and distribution of LMC and SMC 
clusters and associations. Finally, as far as our scheme is concerned, the next step will be its automation through an interactive program treating the spectra in digital form.

\section{Acknowledgements}

We would like to express our sincere thanks to the $1.2 \mathrm{~m}$ UKST Unit for loan of the observational material.

\section{References}

Dapergolas, A., Kontizas, E. and Kontizas, M., 1986. Astron. Astrophys. Suppl. Ser., 65, 283.

Hantzios, P.A., Dapergolas, A., Kontizas, M. and Kontizas, E., 1993. 'Memoirs of the National Observatory of Athens', Series I, Astronomy, \#35.

Kontizas, E., Dapergolas, A. and Kontizas, M., 1986. Astron. Astrophys. Suppl. Ser., 66, 51.

Savage, A., Waldron, D., Morgan, D.H.., Tritton, S.B., Cannon, R., Dave, J., Bruck, R.M., Beard, S.M. and Palmer, B.J., 1985. 'The UKST Objective Prisms' (Publications of the R.O.E.).

Seitter, W.C., 1975. 'Atlas of Obj. Prism Spectra', Bonner Spectral Atlas, Ferd. Dummlers Verlag, Bonn. 